\title{
OBSERVATION OF THERMAL EFFECTS ON THE LEP WIRE SCANNERS
}

\author{
J. Camas, C. Fischer, J.J. Gras, R. Jung, J. Koopman \\ CERN, European Organisation for Nuclear Research \\ CH 1211 Geneva 23, Switzerland
}

\begin{abstract}
A new wire scanner was installed in LEP for the 1994 run. It incorporates an improved mechanical design for the wire movement and can accept three mechanisms, two for horizontal and one for vertical scans. Wires of different materials and of various diameters were fixed to new ceramic forks. Viewing ports were installed opposite each wire to allow the directobservation of the wires with a TV camera during a scan. Recordings of the wire resistance and of the light emitted by the wire were made during scans. These observations have provided the first experimental evidence of the various wire heating mechanisms by the beams. The heating due to coupling to the electro-magnetic field generated by the beam exceeds the heating due to particle collisions. An evaluation is made on the behaviour of Carbon and Quartz wires. Conclusions are drawn for defining a safe operating regime.
\end{abstract}

\section{INTRODUCTION}

Wire destruction has always been a major concern with wire scanners, particularly with instruments installed on circular lepton machines like LEP, where heating of the wire by the RF fields generated by the short lepton bunches adds to the energy deposited locally by the intercepted particles. In the case of LEP, the RF heating is in fact the major cause of wire destruction. Several studies have been made since the LEP start-up, the latest with direct observation of the wire with a TV camera, in order to better understand the wire heating process so that the beam current limit for safe operation of the instrument could be increased. This is important as the wire scanners are the absolute calibration device for the synchrotron light instruments used continuously in operation [1].

\section{HARDWARE EVOLUTION AND STATUS}

The original problems and the evolution of the LEP wire scanners are described in Ref. [2,3]. The original forks were part of a common design for LEP and SPS. Similar setups in the SPS [4] operate with beams of up to $1.6 \times 10^{13} \mathrm{p}$, well above the LEP nominal intensity of $6 \mathrm{~mA}$, i.e. $3.3 \times 10^{12}$ leptons. The vacuum tanks supporting the mechanisms were designed to achieve as low an impedance as possible. The calculated longitudinal loss factor of the tank is equal to $0.1 \mathrm{~V} / \mathrm{pC}$, of which $0.03 \mathrm{~V} / \mathrm{pC}$ come from the horizontal wire parking cavity. For the originally designed wire scanner a temperature rise of $1420^{\circ}$ was estimated from energy deposition in the Carbon fibre at a current of $6 \mathrm{~mA}$ [5]. A refined estimate considering density effects within the wire material brought this down to $1050^{\circ}$. In the first full year of operation, wires were destroyed at much lower currents. On one occasion, the Carbon wire was replaced by a $50 \mu \mathrm{m}$ Beryllium wire, which was analysed after its destruction [2]. The analysis showed that the wire had melted towards the extremities, far from the beam impact, at a beam current well below the nominal one. RF energy deposition was suspected and wire resistance measurements were initiated to estimate the average wire temperature increase during a scan. These scans confirmed the RF heating hypothesis, demonstrating the dominance of magnetic coupling to the wire when far from the beam and of electric field coupling when approaching the beam, well before the wire encounters a significant amount of leptons [2]. Two levels of constant temperature were measured when far from the beam, see Fig.1. From the parking position to about $15 \mathrm{~mm}$ from the beam, a temperature of $520 \% \mathrm{~mA}$ was recorded. After the beam traversal, when the beam passed through the loop comprising of the fork and the wire, a second level of $1000^{\circ} / \mathrm{mA}$ was estimated.

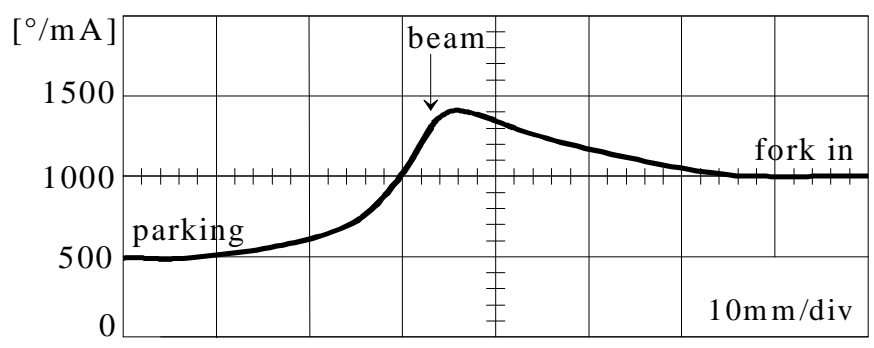

Fig. 1: Wire Temperature evolution during a scan with the original tank and fork set-up at a wire speed of $0.2 \mathrm{~m} / \mathrm{s}$.

These levels were thought to result from magnetic coupling of the fork/wire loop to the wakefield in the tank for the first case, and to the magnetic field generated by the beam for the other case. This coupling was strongly reduced by modifying the fork construction by breaking a parasitic loop [3]: see "old\#2" fork in Table 1. Temperature levels of $230 \% \mathrm{~mA}$ and $530 \% \mathrm{~mA}$ were then measured. The parking temperature was even reduced to $80 \% \mathrm{~mA}$ for the vertical wires which were better shielded against RF fields. The temperature peak in Fig. 1 corresponds to coupling to the beam electric field and depends on the wire length and on the RF spectrum of the bunch. These hypotheses agreed with observations made where the wire temperature changed at constant current when the bunch length was changed [3]. The beam current limit could then be increased to circulating 
beams of $3 \mathrm{~mA}$ at a wire speed of $0.5 \mathrm{~m} / \mathrm{s}$. After analysis, it was concluded that the current limit could only be further increased by redesigning the vacuum tank to lower the loss factor and the supporting fork to break any remaining current loop and by choosing the wire length below wavelengths present in the beam spectrum. Isolating materials which prevent RF currents from heating the wire were also considered. A new vacuum tank having the elliptical shape of the adjacent chambers and with small parking cavities was designed. Its loss factor is $0.04 \mathrm{~V} / \mathrm{pC}$ per horizontal parking cavity. Two horizontal and one vertical wire ports were fitted to the tank. They provided the possibility to make scans along three directions (by using inclined wires) and to test various wire materials. Also incorporated were viewing ports to observe the wires with TV cameras: Fig 2.

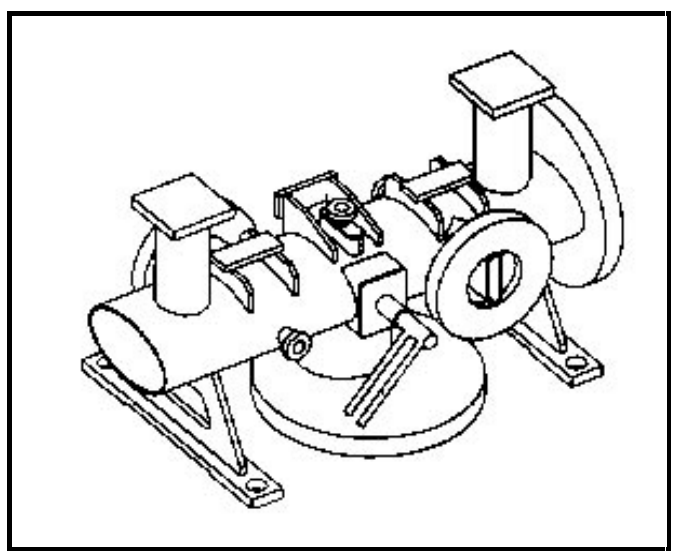

Fig. 2: CAD view of the New Wire Scanner Vacuum Tank: small viewing ports can be seen at the left and top, and flanges for the wire mechanisms at the bottom and right.

The forks were redesigned and made entirely of ceramics, with the possibility to stretch three wires spaced by $6 \mathrm{~mm}$ across the aperture: Fig. 3. Carbon wires of 8 and 36 $\mu \mathrm{m}$ and Quartz wires of $33 \mu \mathrm{m}$ were installed and tested.

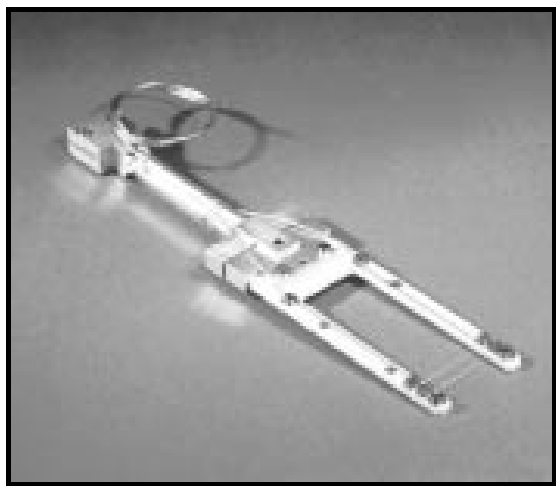

Fig. 3: The new ceramic fork with three wires.

\section{TEMPERATURE MEASUREMENT}

The temperature is estimated from resistance variations. The Carbon wire is put in the feedback loop of an operational amplifier tuned for zero output with no beam. This amplifier output is acquired during the scans together with the beam profile measured by a scintillator and the wire position measured with a high precision optical ruler [2]. The results are displayed in real time and saved. The live TV signal is observed during a scan and connected to a video recorder for off-line digitisation. The wire resistivity variation with temperature was estimated. Measurements in an oil bath at low temperature and with a pyrometer at high temperature have been performed. The results are plotted in Fig. 4. Both show a similar negative temperature coefficient. Absolute measurements are difficult, but estimations of temperature variations should be satisfactory up to $1800^{\circ} \mathrm{C}$.

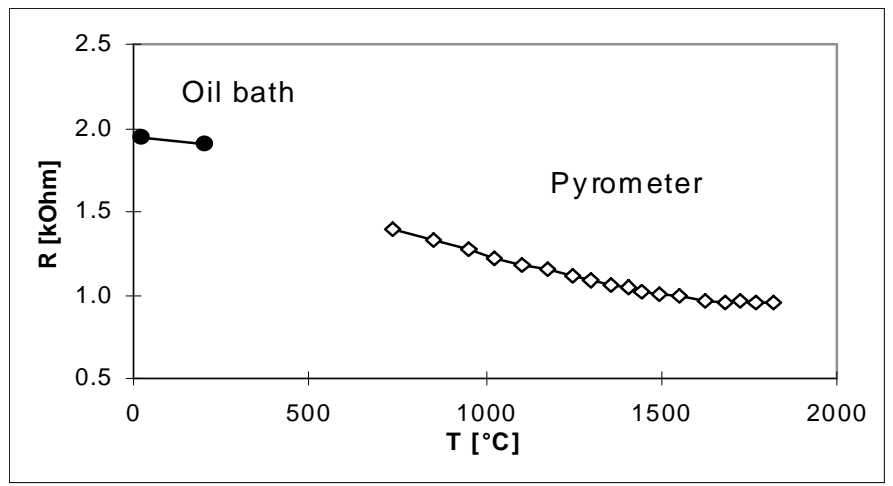

Fig 4: Measured wire resistance variations with temperature.

\section{RESULTS}

The temperature measurements during a scan, using the same beam, with old and new tanks and forks, short and long wires show the improvements achieved: Table 1.

Table 1: Normalised average temperature increase of wires

\begin{tabular}{|c|c|c|c|c|c|}
\hline Location & Tank & Fork & Wire length & $\Delta$ Tpark. & $\Delta$ T max \\
\hline $139 / \mathrm{V} \mathrm{e}^{-}$ & old & old\#2 & $56 \mathrm{~mm}$ & $80 \% \mathrm{~mA}$ & $620 \% \mathrm{~mA}$ \\
\hline $140 / \mathrm{H} \mathrm{e}^{-}$ & old & old\#2 & $56 \mathrm{~mm}$ & $200 \% \mathrm{~mA}$ & $640 \% \mathrm{~mA}$ \\
\hline $160 / \mathrm{H} \mathrm{e}^{+}$ & old & old\#2 & $29 \mathrm{~mm}$ & $30 \% \mathrm{~mA}$ & $520 \% \mathrm{~mA}$ \\
\hline $161 / \mathrm{V} \mathrm{e}^{+}$ & new & new & 3 of $29 \mathrm{~mm}$ & $20 \% \mathrm{~mA}$ & $500 \% \mathrm{~mA}$ \\
\hline
\end{tabular}

As a consequence of the new design, the wire heating in the parking position is now negligible and the maximum temperature reached during a scan has decreased confirming the heating by coupling to the electro-magnetic fields. Recordings show again a temperature increase well before reaching the beam and a slow decrease after the traversal. When the wire crosses the beam, the slope of the temperature curve is only slightly changed, indicating that the global temperature increase due to the beam-wire collision is small (Fig. 5). This is consistent with the expected temperature rise for collision losses of $175 \% \mathrm{~mA}$, see Section II, on the wire portion hit by the beam ( $1 \mathrm{~mm}$ or $2 \mathrm{~mm}$ for the $\mathrm{H}$ or $\mathrm{V}$ scans) and the induced average resistance variation of the full wire length $(29 \mathrm{~mm}$ or $56 \mathrm{~mm})$. The latter is very small (in the 
ratio of the respective lengths when assuming no conduction) and is much lower than the observed temperature increase when the wire passes inside the beam distribution.
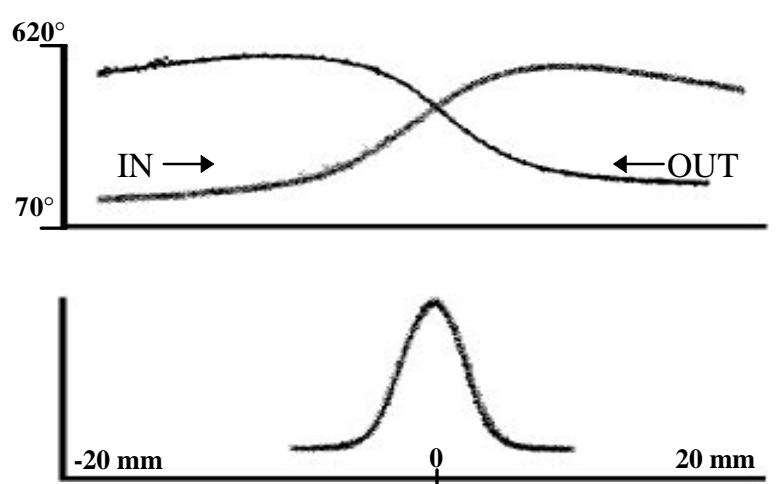

Fig. 5: Resistance/Temperature curves and beam profiles during a full IN and OUT horizontal scan of a $1.2 \mathrm{~mA}$ beam.

The video scans confirm the RF heating of the wire. Whatever the position, except perhaps when passing through the beam where the CCD camera saturates, the extremities of the wire are the hottest: Fig 6 . The wire average temperature as estimated from the resistance measurement underestimates the peak temperature by more than a factor of two. Similar temperature profiles are obtained by simulations when considering the wire as a dipole in the RF fields generated by the beam along its direction. They confirm also that the long $56 \mathrm{~mm}$ wire will heat up more than the shorter $29 \mathrm{~mm}$ wire. This dipole type temperature profile is globally helping the wire survival as it has a minimum at the centre where the wire is heated by the collision with the beam. These observations were made primarily with the fork holding three wires: two carbon wires of 8 and $36 \mu \mathrm{m}$, and a quartz wire of $33 \mu \mathrm{m}$. The $8 \mu \mathrm{m}$ carbon wire broke at a current of about 1.5 $\mathrm{mA}$ and the $36 \mu \mathrm{m}$ one at $4.3 \mathrm{~mA}$, well above previous limits. The average maximum wire temperature during the scan was in this case about $1750^{\circ}$. The Quartz wire survived these currents despite its lower melting temperature and is still in use. No light emission during scans has so far been seen from either of the Quartz wires.

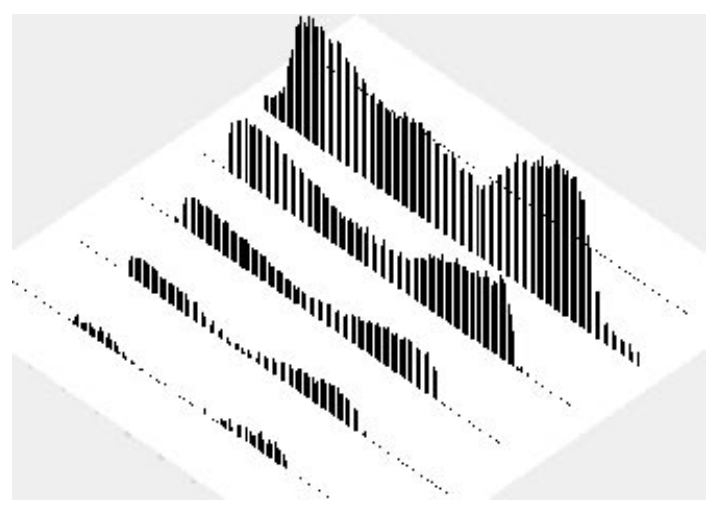

Fig 6: Light emission profile from the $36 \mu \mathrm{m}$ Carbon wire when approaching a LEP beam of $1.67 \mathrm{~mA}$ total intensity. The profiles are taken every $20 \mathrm{~ms}$ at a wire speed of $0.5 \mathrm{~m} / \mathrm{s}$.

\section{CONCLUSION}

Integrated wire resistance measurements and the observation of light emission from the scanning wires confirm that the major mechanism for wire destruction in lepton storage rings is by RF fields. These fields act on the full wire length far away from the encounter of beam and wire. The induced temperature increase is more than twice that of the estimated heating from beam energy deposition in the central part of the wire. The acceptable current limits are hence reduced for conducting materials like Carbon, and are even lower for Beryllium. These phenomena impose design constraints which can be summarised as follows:

- vacuum tanks with as low loss factors as possible

- wire parking locations connected to the beam pipe with sufficient attenuation for the beam RF spectrum

- wires as short as possible, with a length preferably below the two sigma limit of the bunch wavelength spectrum

- wire supporting forks which effectively break any loop passing through the wire for the magnetic field coupling.

The latest design lowered the integrated temperature increase during a scan to $500 \% \mathrm{~mA}$, with wire ends more than a factor of two hotter than the centre. This sets the maximum current limit at $3.7 \mathrm{~mA}$, with a safe current level at $2 \mathrm{~mA}$. Wires made of isolating material, here Quartz, have given the best results for maximum beam current. More experience has to be accumulated to assess their susceptibility to the strong electrical fields generated by high intensity beams, but they withstood scans of beams of more than $4 \mathrm{~mA}$, i.e. more than twice the safe threshold value for Carbon fibres.

Monitoring the thermal behaviour of the wires through resistance measurement and direct video observation has been extremely helpful.

\section{ACKNOWLEDGEMENTS}

The new tank was designed by C. Menot and manufactured by the CERN workshops. The loss factors were calculated by P. Valentin. G. Ferioli, J. Mann and J. Provost helped with the resistance and video measurements and J.M. Vouillot with the illustrations.

Their contributions are greatly acknowledged.

\section{REFERENCES}

[1] P. Castro et al.: Comparative precision emittance measurements in LEP, Proc. of EPAC 94, London, 1994

[2] B. Bouchet et al.: Wire scanners at LEP, Proc. of the 1991 IEEE Acc. Conf., San Francisco, 1991

[3] J. Camas et al.: High resolution measurements of lepton transverse distributions with the LEP wire scanners, Proc. of the 1993 IEEE Acc. Conf., Washington D.C., 1993 
[4] A. Burns et al.: Wire scanner news from the CERNSPS, Proc. of the 1989 IEEE Acc. Conf., Chicago, 1989

[5] C. Fischer et al.: Studies of fast wire scanners for LEP, proc. of the 1988 EPAC, Rome, 1988 